\title{
Schultz and Modified Schultz Polynomials of Vertex Identification Chain for Square and Complete Square Graphs
}

\author{
Mahmood M. Abdullah, Ahmed M. Ali \\ College of Computer Sciences and Mathematics: Mathematics, University of Mosul, Mosul, Iraq \\ Email:Mmadain7@gmail.com, ahmedgraph@uomosul.edu.iq
}

How to cite this paper: Abdullah, M.M. and Ali, A.M. (2020) Schultz and Modified Schultz Polynomials of Vertex Identification Chain for Square and Complete Square Graphs. Open Access Library Journal, 7: e6309.

https://doi.org/10.4236/oalib.1106309

Received: April 8, 2020

Accepted: May 18, 2020

Published: May 21, 2020

Copyright $\odot 2020$ by author(s) and Open Access Library Inc.

This work is licensed under the Creative

Commons Attribution International

License (CC BY 4.0).

http://creativecommons.org/licenses/by/4.0/

\begin{abstract}
In this paper, we find the polynomials, indices and average distance for Schultz and modified Schultz of vertex identification chain for 4-cycle and 4cycle complete.
\end{abstract}

Subject Areas

Discrete Mathematics, Graph Theory, Combinatory

\section{Keywords}

Schultz, Modified Schultz, Polynomials, Indices, Chain of Vertex Identification Graphs

\section{Introduction}

Given the importance of topological evidence, which can be deduced from the polynomial by finding the derivative with respect to a specific variable and then compensating for this variable by one value, therefore we have in this paper found the polynomial of Schultz and modified Schultz for a chain of special graphs which is the 4-cycle and 4-cycle complete by identification symmetrical vertices.

In this paper, we can refer to the basic concepts in graph theory and topological indices to the references [1] [2] [3]. Let $G=(V, E)$ be a simple connected graph without loop and multi-edges, where $V=V(G)$ and $E=E(G)$ be a set of vertices and edges of respect to a graph $G$. The distance between any two vertices of $V(G)$ is the shortest path between them which denoted its by $d(u, v)$, $u, v \in V(G)$ and the maximum distance between any two vertices in $G$ is called 
the diameter graph $G$, that is: $\delta=\max _{u, v \in V(G)}\{d(u, v)\}$. The degree of vertex $u$ in a graph $G$ is the number of the edges which incident on $u$ and denoted by $\operatorname{deg} u$.

Introduced Schultz index by Schultz in 1989 [4] and in 1997 Klavžar and Gutman were defined the modified Schultz index [5]. The Schultz index $\operatorname{Sc}(G)$ and the modified Schultz index $S c^{*}(G)$ are have defined as:

$$
\begin{aligned}
& S c(G)=\sum_{\{u, v\} \subseteq V(G)}(\operatorname{deg} v+\operatorname{deg} u) d(u, v) . \\
& S c^{*}(G)=\sum_{\{u, v\} \subseteq V(G)}(\operatorname{deg} v \cdot \operatorname{deg} u) d(u, v) .
\end{aligned}
$$

The Schultz and modified Schultz polynomials are have defined respectively as:

$$
\begin{aligned}
& S c(G ; x)=\sum_{\{u, v\} \subseteq V(G)}(\operatorname{deg} v+\operatorname{deg} u) x^{d(u, v)} . \\
& S c^{*}(G ; x)=\sum_{\{u, v\} \subseteq V(G)}(\operatorname{deg} v \cdot \operatorname{deg} u) x^{d(u, v)} .
\end{aligned}
$$

From these polynomials can obtain:

1) The Schultz index:

$$
S c(G)=\left.\frac{\mathrm{d}}{\mathrm{d} x}(S c(G ; x))\right|_{x=1} .
$$

2) The modified Schultz index:

$$
S c^{*}(G)=\left.\frac{\mathrm{d}}{\mathrm{d} x}\left(S c^{*}(G ; x)\right)\right|_{x=1} .
$$

3) The average distance of Schultz:

$$
\overline{S c}(G)=2 S c(G) / p(G)(p(G)-1)
$$

4) The average distance of modified Schultz:

$$
\overline{S c^{*}}(G)=2 S c^{*}(G) / p(G)(p(G)-1) .
$$

where $\frac{\mathrm{d}}{\mathrm{d} x}$ is represent the derivative w.r.t. $x$ and $p(G)$ is the order of $G$.

There are many recent papers on polynomials and indices for Schultz and modified Schultz, see to references [6] [7] [8] and there are applications on Schultz and modified Schultz in chemistry, see to references [9] [10] [11].

Let $D_{k}(r, h)$ be the set of all $(u, v)$ of $G$ which distance between $u$ and $v$ is $k$ such that $\operatorname{deg} u=r$ and $\operatorname{deg} v=h$ and $\left|D_{k}(G)\right|$ is the number of pairs $(u, v)$ of $G$ that are distance $k$ " $D(G, k)$ ".

From clearly that $\sum_{k=1}^{\operatorname{diam}(G)}\left|D_{k}(G)\right|=p(G)(p(G)-1) / 2$.

\section{The Vertex-Identification Chain (VIC)-Graphs}

Let $\left\{G_{1}, G_{2}, \cdots, G_{n}\right\}$ be a set of pairwise disjoint graphs with vertices $u_{i}, v_{i} \in V\left(G_{i}\right), \quad i=1,2, \cdots, n, n \geq 2$, then the vertex-identification chain graph $C_{v}\left(G_{1}, G_{2}, \cdots, G_{n}\right) \equiv C_{v}\left(G_{1}, G_{2}, \cdots, G_{n}: v_{1} \cdot u_{2} ; v_{2} \cdot u_{3} ; \cdots ; v_{n-1} \cdot u_{n}\right)$ of $\left\{G_{i}\right\}_{i=1}^{n}$ with respect to the vertices $\left\{v_{i}, u_{i+1}\right\}_{i=1}^{n-1}$ is the graph obtained from the graphs $G_{1}, G_{2}, \cdots, G_{n}$ by identifying the vertex $v_{i}$ with the vertex $u_{i+1}$ for all $i=1,2, \cdots, n-1$. (See Figure 1): 

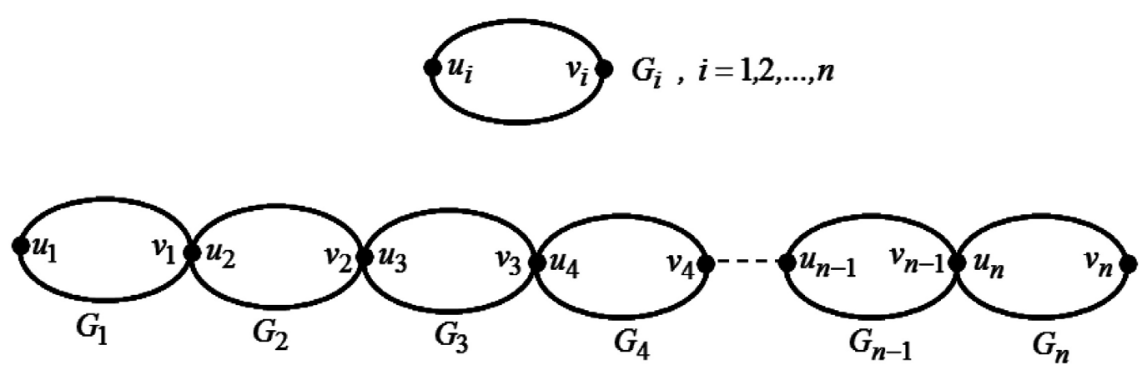

Figure 1. The graph $C_{v}\left(G_{1}, G_{2}, \cdots, G_{n}\right)$.

Some Properties of Graph $C_{v}\left(G_{1}, G_{2}, \cdots, G_{n}\right)$ :

1) $p\left(C_{v}\left(G_{1}, G_{2}, \cdots, G_{n}\right)\right)=\sum_{i=1}^{n} p\left(G_{i}\right)-(n-1)$.

2) $q\left(C_{v}\left(G_{1}, G_{2}, \cdots, G_{n}\right)\right)=\sum_{i=1}^{n} q\left(G_{i}\right)$.

3) $n \leq \operatorname{diam}\left(C_{v}\left(G_{1}, G_{2}, \cdots, G_{n}\right)\right) \leq \sum_{i=1}^{n} \operatorname{diam}\left(G_{i}\right)$.

The equality of lower bound is satisfied at $K_{2}$ but the upper bound is satisfied at path graph.

If $G_{i} \equiv H_{p}$, for all $1 \leq i \leq n$, where $H_{p}$ is a connected graph of order $p$, we denoted $C_{v}\left(H_{p}, H_{p}, \cdots, H_{p}\right)$ by $C_{v}\left(H_{p}\right)_{n}$.

\subsection{Schultz and Modified Schultz of $C_{v}\left(C_{4}\right)_{p}$}

From Figure 2, we note that $p\left(C_{v}\left(C_{4}\right)_{p}\right)=3 p+1, q\left(C_{v}\left(C_{4}\right)_{p}\right)=4 p$ and $\operatorname{diam}\left(C_{v}\left(C_{4}\right)_{p}\right)=2 p$, for all $1 \leq i, j \leq p, i \neq j, 2 \leq h, k \leq p, h \neq k$, then we have:

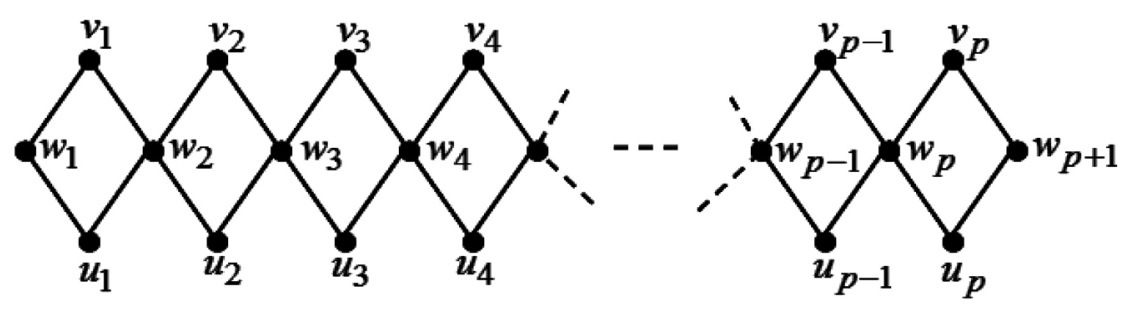

Figure 2. The graph $C_{v}\left(C_{4}\right)_{p}$.

Table 1. The vertices degrees of the graph $C_{v}\left(C_{4}\right)_{p}$.

\begin{tabular}{|c|c|c|c|c|c|}
\hline$\gamma^{+}$ & $\operatorname{deg} u_{i}=2$ & $\operatorname{deg} v_{i}=2$ & $\operatorname{deg} w_{1}=2$ & $\operatorname{deg} w_{h}=4$ & $\operatorname{deg} w_{p+1}=2$ \\
\hline $\operatorname{deg} u_{j}=2$ & & & & & \\
\hline $\operatorname{deg} v_{j}=2$ & & & & & \\
\hline $\operatorname{deg} w_{1}=2$ & & & & & \\
\hline$d e g w_{k}=4$ & & & & & \\
\hline $\operatorname{deg} w_{p+1}=2$ & & & & & \\
\hline
\end{tabular}


Theorem 2.1.1: For $p \geq 2$, then we have:

1) $S c\left(C_{v}\left(C_{4}\right)_{p} ; x\right)=8(3 p-1) x+4(7 p-5) x^{2}+4 \sum_{k=3}^{2 p}(6 p-3 k+1) x^{k}$.

2) $S c^{*}\left(C_{v}\left(C_{4}\right)_{p} ; x\right)=16(2 p-1) x+4(9 p-8) x^{2}+16 \sum_{k=3}^{2 p-1}(2 p-k) x^{k}+4 x^{2 p}$.

Proof: For all $p \geq 4$ and every two vertices $u, v \in V\left(C_{v}\left(C_{4}\right)_{p}\right)$, there is $d(u, v)=k, 1 \leq k \leq 2 p$, then obviously, $\sum_{i=1}^{2 p}\left|D_{i}\right|=\frac{3 p(3 p+1)}{2}$. We will have six partitions for proof:

P1. If $d(u, v)=1$, then $\left|D_{1}\right|=4 p=q\left(C_{v}\left(C_{4}\right)_{p}\right)$ and we have two subsets of it:

P1.1. $\left|D_{1}(2,2)\right|=\left|\left\{\left(u_{1}, w_{1}\right),\left(u_{p}, w_{p+1}\right),\left(v_{1}, w_{1}\right),\left(v_{1}, w_{p}\right)\right\}\right|=4$.

P1.2. $\left|D_{1}(2,4)\right|=\left|\left\{\left(u_{i-1}, w_{i}\right),\left(v_{i-1}, w_{i}\right),\left(u_{i}, w_{i}\right),\left(v_{i}, w_{i}\right): 2 \leq i \leq p\right\}\right|=4(p-1)$.

P2. If $d(u, v)=2$, then $\left|D_{2}\right|=6 p-4$ and we have three subsets of it:

P2.1. $\left|D_{2}(2,2)\right|$

$$
\begin{aligned}
& =\left|\left\{\left(u_{i}, u_{i+1}\right),\left(u_{i}, v_{i+1}\right),\left(v_{i}, v_{i+1}\right),\left(v_{i}, u_{i+1}\right): 1 \leq i \leq p-1\right\} \cup\left\{\left(u_{i}, v_{i}\right): 1 \leq i \leq p\right\}\right| \\
& =5 p-4 .
\end{aligned}
$$

P2.2. $\left|D_{2}(2,4)\right|=\left|\left\{\left(w_{1}, w_{2}\right),\left(w_{p+1}, w_{p}\right)\right\}\right|=2$.

P2.3. $\left|D_{2}(4,4)\right|=\left|\left\{\left(w_{i}, w_{i+1}\right): 2 \leq i \leq p-1\right\}\right|=p-2$.

P3. If $d(u, v)=k$, then $\left|D_{k}\right|=9 p+\frac{1}{2} k+3$, and we have:

1) If $k$ is an odd, $3 \leq k \leq 2 p-3$, then $\left|D_{k}\right|=4 p-2 k+2$, and we have two subsets of it:

P3.1. $\left|D_{k}(2,2)\right|=\left|\left\{\left(w_{1}, u_{k-\frac{k-1}{2}}\right),\left(w_{1}, v_{k-\frac{k-1}{2}}\right),\left(w_{1}, u_{k-\frac{k-1}{2}}\right),\left(w_{1}, v_{k-\frac{k-1}{2}}\right)\right\}\right|=4$.

P3.2.

$$
\begin{aligned}
\left|D_{k}(2,4)\right| & =\left|\left\{\left(u_{i+\frac{k-1}{2}}, w_{i}\right),\left(v_{i+\frac{k-1}{2}}, w_{i}\right),\left(u_{i-1}, w_{i+\frac{k-1}{2}}\right),\left(v_{i-1}, w_{i+\frac{k-1}{2}}\right): 2 \leq i \leq p-\frac{k-1}{2}\right\}\right| \\
& =4\left(p-\frac{k-1}{2}-1\right) .
\end{aligned}
$$

2) If $k$ is an even, $4 \leq k \leq 2 p-4$, then $\left|D_{k}\right|=5 p+\frac{5}{2} k+1$, and we have three subsets of it:

P3.3. $\left|D_{k}(2,2)\right|=\left|\left\{\left(u_{i}, u_{i+\frac{k}{2}}\right),\left(u_{i}, v_{i+\frac{k}{2}}\right),\left(v_{i}, v_{i+\frac{k}{2}}\right),\left(v_{i}, u_{i+\frac{k}{2}}\right): 1 \leq i \leq p-\frac{k}{2}\right\}\right|$

$$
=4\left(p-\frac{k}{2}\right) \text {. }
$$

P3.4. $\left|D_{k}(2,4)\right|=\left|\left\{\left(w_{1}, w_{1+\frac{k}{2}}\right),\left(w_{p+1}, w_{p-\frac{k}{2}+1}\right)\right\}\right|=2$.

P3.5. $\left|D_{k}(4,4)\right|=\left|\left\{\left(w_{i}, w_{i+\frac{k}{2}}\right): 2 \leq i \leq p-\frac{k}{2}\right\}\right|=p-\frac{k}{2}-1$. 
P4. If $d(u, v)=2 p-2$, then $\left|D_{2 p-2}\right|=6$, and we have two subsets of it:

P4.1. $\left|D_{2 p-2}(2,2)\right|=\left|\left\{\left(u_{1}, u_{p}\right),\left(v_{1}, v_{p}\right),\left(u_{1}, v_{p}\right),\left(v_{1}, u_{p}\right)\right\}\right|=4$.

P4.2. $\left|D_{2 p-2}(2,4)\right|=\left|\left\{\left(w_{1}, w_{p}\right),\left(w_{p+1}, w_{2}\right)\right\}\right|=2$.

P5. If $d(u, v)=2 p-1$, then $\left|D_{2 p-1}\right|=4$, and we have one subset of it:

$$
\left|D_{2 p-1}(2,2)\right|=\left|\left\{\left(w_{1}, u_{p}\right),\left(w_{1}, v_{p}\right),\left(w_{p+1}, u_{1}\right),\left(w_{p+1}, v_{1}\right)\right\}\right|=4 .
$$

P6. If $d(u, v)=2 p$, then $\left|D_{2 p}\right|=1$, and we have one subset of it:

$$
\left|D_{2 p}(2,2)\right|=\left|\left\{\left(w_{1}, w_{p+1}\right)\right\}\right|=1 .
$$

Hence, from P1 to P6 and Table 1, we get:

$$
\begin{aligned}
& S c\left(C_{v}\left(C_{4}\right)_{p} ; x\right)=\{4(4)+6(4(p-1))\} x+\{4(5 p-4)+6(2)+8(p-2)\} x^{2} \\
& +\left\{\sum_{k=3}^{2 p-3}\left\{4(4)+6\left(4\left(p-\frac{k-1}{2}-1\right)\right)\right\} x^{k}, \quad k\right. \text { is an odd } \\
& +\sum_{k=4}^{2 p-4}\left\{4\left(4\left(p-\frac{k}{2}\right)\right)+6(2)+8\left(p-\frac{k}{2}-1\right)\right\} x^{k}, k \text { is an even } \\
& +\{4(4)+6(2)\} x^{2 p-2}+\{4(4)\} x^{2 p-1}+\{4(1)\} x^{2 p} \\
& =8(3 p-1) x+4(7 p-5) x^{2}+4 \sum_{k=3}^{2 p}(6 p-3 k+1) x^{k} .
\end{aligned}
$$

And,

$$
\begin{aligned}
& S^{*}\left(C_{v}\left(C_{4}\right)_{p} ; x\right)=\{4(4)+8(4(p-1))\} x+\{4(5 p-4)+8(2)+16(p-2)\} x^{2} \\
& +\left\{\sum_{k=3}^{2 p-3}\left\{4(4)+8\left(4\left(p-\frac{k-1}{2}-1\right)\right)\right\} x^{k}, \quad k \text { is an odd },\right. \\
& \sum_{k=4}^{2 p-4}\left\{4\left(4\left(p-\frac{k}{2}\right)\right)+8(2)+16\left(p-\frac{k}{2}-1\right)\right\} x^{k}, k \text { is an even } \\
& +\{4(4)+8(2)\} x^{2 p-2}+\{4(4)\} x^{2 p-1}+\{4(1)\} x^{2 p} \\
& =16(2 p-1) x+4(9 p-8) x^{2}+16 \sum_{k=3}^{2 p-1}(2 p-k) x^{k}+4 x^{2 p} .
\end{aligned}
$$

By simple, we can calculate:

1) $\operatorname{Sc}\left(C_{v}\left(C_{4}\right)_{2} ; x\right)=40 x+36 x^{2}+16 x^{3}+4 x^{4}$.

$$
S c^{*}\left(C_{v}\left(C_{4}\right)_{2} ; x\right)=48 x+40 x^{2}+16 x^{3}+4 x^{4} \text {. }
$$

2) $S c\left(C_{v}\left(C_{4}\right)_{3} ; x\right)=64 x+64 x^{2}+40 x^{3}+28 x^{4}+16 x^{5}+4 x^{6}$.

$$
S c^{*}\left(C_{v}\left(C_{4}\right)_{3} ; x\right)=80 x+76 x^{2}+48 x^{3}+32 x^{4}+16 x^{5}+4 x^{6} .
$$

Corollary 2.1.2: For $p \geq 2$, then we have:

1) $S c\left(C_{v}\left(C_{4}\right)_{p}\right)=8 p\left(2 p^{2}+p+1\right)$.

2) $S c^{*}\left(C_{v}\left(C_{4}\right)_{p}\right)=\frac{32}{3} p\left(2 p^{2}+1\right)$.

Corollary 2.1.3: For $p \geq 2$, then we have:

1) $\overline{S c}\left(C_{v}\left(C_{4}\right)_{p}\right)=\frac{16}{27}(6 p+1+8 /(3 p+1))$. 
2) $\overline{S c^{*}}\left(C_{v}\left(C_{4}\right)_{p}\right)=\frac{128}{81}(3 p-1+11 / 2(3 p+1))$.

\subsection{Schultz and Modified Schultz of $C_{v}\left(K_{4}\right)_{p}$}

From Figure 3, we note that $p\left(C_{v}\left(K_{4}\right)_{p}\right)=3 p+1, q\left(C_{v}\left(K_{4}\right)_{p}\right)=6 p$ and $\operatorname{daim}\left(C_{v}\left(K_{4}\right)_{p}\right)=p$, for all $1 \leq i, j \leq p, \quad i \neq j, \quad 2 \leq h, k \leq p, h \neq k$, then we have:
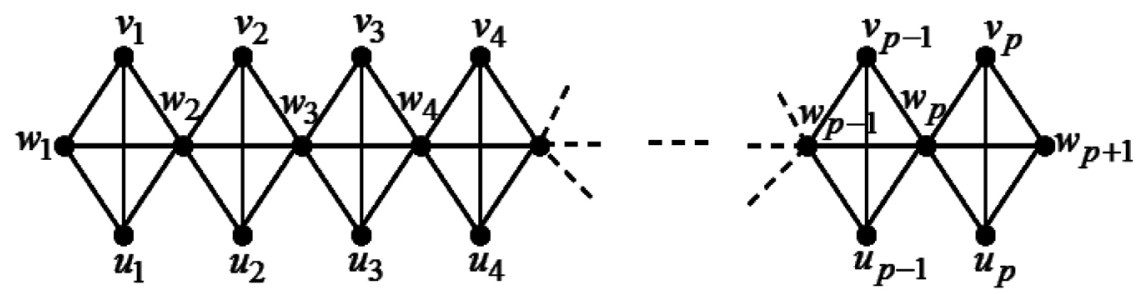

Figure 3. The graph $C_{v}\left(K_{4}\right)_{p}$.

Table 2. The vertices degrees of the graph $C_{v}\left(K_{4}\right)_{p}$.

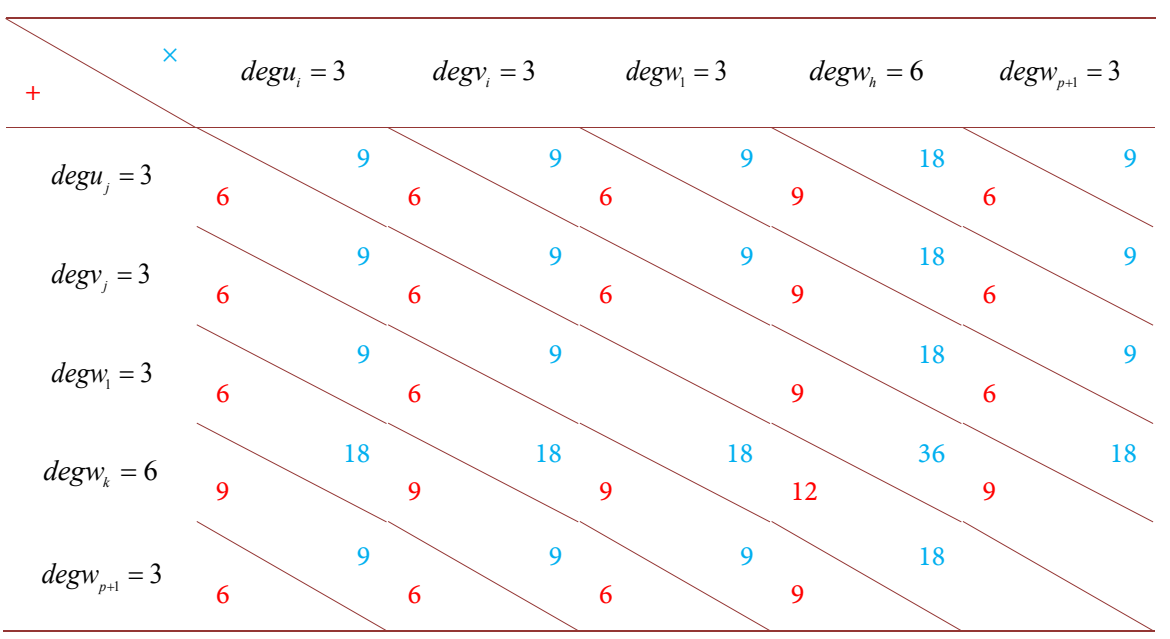

Theorem 2.2.1: for $p \geq 3$, then we have:

1) $S c\left(C_{v}\left(K_{4}\right)_{p} ; x\right)=18(3 p-1) x+18 \sum_{k=2}^{p}(4 p-4 k+3) x^{k}$.

2) $S c^{*}\left(C_{v}\left(K_{4}\right)_{p} ; x\right)=9(13 p-8) x+72 \sum_{k=2}^{p-1}(2 p-2 k+1) x^{k}+81 x^{p}$.

Proof: For all $p \geq 4$, and every vertex $u, v \in V\left(C_{v}\left(K_{4}\right)_{p}\right)$ there is $d(u, v)=k, 1 \leq k \leq p$, then obviously, $\sum_{i=1}^{2 p}\left|D_{i}\right|=\frac{3 p(3 p+1)}{2}$. We will have four partitions for proof:

P1. If $d(u, v)=1$, then $\left|D_{1}\right|=6 p=q\left(C_{v}\left(K_{4}\right)_{p}\right)$ and we have three subsets of it: P1.1. $\left|D_{1}(3,3)\right|=\left|\left\{\left(u_{i}, v_{i}\right): 1 \leq i \leq p\right\} \bigcup\left\{\left(u_{1}, w_{1}\right),\left(v_{1}, w_{1}\right),\left(u_{p}, w_{p+1}\right),\left(v_{p}, w_{p+1}\right)\right\}\right|$

$$
=p+4 \text {. }
$$


P1.2. $\left|D_{1}(3,6)\right|=\mid\left\{\left(u_{i}, w_{i}\right),\left(u_{i}, w_{i+1}\right),\left(v_{i}, w_{i}\right),\left(v_{i}, w_{i+1}\right): 2 \leq i \leq p-1\right\}$

$$
\begin{aligned}
& \cup\left\{\left(u_{p}, w_{p}\right),\left(u_{1}, w_{2}\right),\left(v_{p}, w_{p}\right),\left(v_{1}, w_{2}\right),\left(w_{1}, w_{2}\right),\left(w_{p}, w_{p+1}\right)\right\} \mid \\
= & 4 p-2 .
\end{aligned}
$$

P1.3. $\left|D_{1}(6,6)\right|=\left|\left\{\left(w_{i}, w_{i+1}\right): 2 \leq i \leq p-1\right\}\right|=p-2$.

P2. If $d(u, v)=k, 2 \leq k \leq p-2$, then $\left|D_{k}\right|=9(p-k+1)$ and we have three subsets of it:

P2.1

$$
\begin{gathered}
\left|D_{k}(3,3)\right|=\mid\left\{\left(u_{i}, u_{i+k-1}\right),\left(v_{i}, v_{i+k-1}\right),\left(u_{i}, v_{i+k-1}\right),\left(v_{i}, u_{i+k-1}\right): 1 \leq i \leq p-k+1\right\} \\
\cup\left\{\left(u_{k}, w_{1}\right),\left(u_{p-k+1}, w_{p+1}\right),\left(v_{k}, w_{1}\right),\left(v_{p-k+1}, w_{p+1}\right)\right\} \mid=4(p-k+2) .
\end{gathered}
$$

P2.2.

$$
\begin{aligned}
& \left|D_{k}(3,6)\right| \\
= & \mid\left\{\left(u_{i}, w_{i+k}\right),\left(u_{i+k-1}, w_{i}\right),\left(v_{i}, w_{i+k}\right),\left(v_{i+k-1}, w_{i}\right): 2 \leq i \leq p-k\right\} \\
& \cup\left\{\left(u_{1}, w_{1+k}\right),\left(u_{p}, w_{p-k+1}\right),\left(v_{1}, w_{1+k}\right),\left(v_{p}, w_{p-k+1}\right),\left(w_{1}, w_{1+k}\right),\left(w_{p+1}, w_{p-k+1}\right)\right\} \mid \\
= & 2(2 p-2 k+1) .
\end{aligned}
$$

P2.3. $\left|D_{k}(6,6)\right|=\left|\left\{\left(w_{i}, w_{i+k}\right): 2 \leq i \leq p-k\right\}\right|=p-k-1$.

P3. If $d(u, v)=p-1$, then $\left|D_{p-1}\right|=18$ and we have two subsets of it:

P3.1. $\left|D_{p-1}(3,3)\right|=\left\{\left\{\left(u_{i}, u_{i+p-2}\right),\left(u_{i}, v_{i+p-2}\right),\left(v_{i}, v_{i+p-2}\right),\left(v_{i}, u_{i+p-2}\right): i=1,2\right\}\right.$

$$
\bigcup\left\{\left(u_{2}, w_{p-1}\right),\left(u_{p-1}, w_{1}\right),\left(v_{2}, w_{p+1}\right),\left(v_{p-1}, w_{1}\right)\right\} \mid=12 \text {. }
$$

P3.2. $\left|D_{p-1}(3,6)\right|=\left|\left\{\left(u_{1}, w_{p}\right),\left(u_{p}, w_{2}\right),\left(v_{p}, w_{2}\right),\left(v_{1}, w_{p}\right),\left(w_{1}, w_{p}\right),\left(w_{1}, w_{p}\right)\right\}\right|=6$.

P4. If $d(u, v)=p$, then $\left|D_{p}\right|=9$, and we have one subset of it:

$$
\begin{gathered}
\left|D_{p}(3,3)\right|=\mid\left\{\left(u_{1}, u_{p}\right),\left(u_{1}, w_{p+1}\right),\left(u_{1}, v_{p}\right),\left(v_{1}, w_{p+1}\right),\left(v_{1}, v_{p}\right),\right. \\
\left.\left(u_{p}, w_{1}\right),\left(u_{p}, v_{1}\right),\left(w_{1}, w_{p+1}\right),\left(w_{1}, v_{p}\right)\right\} \mid=9
\end{gathered}
$$

Hence, from $\mathrm{P} 1$ to $\mathrm{P} 4$ and Table 2, we get:

$$
\begin{aligned}
& S c\left(C_{v}\left(K_{4}\right)_{p} ; x\right)=\{6(p+4)+9(4 p-2)+12(p-2)\} x \\
& \quad+\sum_{k=2}^{p-2}\{6(4 p-4 k+8)+9(4 p-4 k+2)+12(p-k-1)\} x^{k} \\
& \quad+\{6(12)+9(6)\} x^{p-1}+\{6(9)\} x^{p} \\
& =18(3 p-1) x+18 \sum_{k=2}^{p}(4 p-4 k+3) x^{k} .
\end{aligned}
$$

And,

$$
\begin{aligned}
& S c^{*}\left(C_{v}\left(K_{4}\right)_{p} ; x\right)=\{9(p+4)+18(4 p-2)+36(p-2)\} x \\
& \quad+\sum_{k=2}^{p-2}\{9(4 p-4 k+8)+18(4 p-4 k+2)+36(p-k-1)\} x^{k} \\
& \quad+\{9(12)+18(6)\} x^{p-1}+\{9(9)\} x^{p} \\
& =9(13 p-8) x+72 \sum_{k=2}^{p-1}(2 p-2 k+1) x^{k}+81 x^{p} .
\end{aligned}
$$


By simply we can calculate:

$$
\begin{aligned}
& S c\left(C_{v}\left(K_{4}\right)_{3} ; x\right)=144 x+126 x^{2}+54 x^{2} . \\
& S c\left(C_{v}\left(K_{4}\right)_{3} ; x\right)=279 x+216 x^{2}+81 x^{2} .
\end{aligned}
$$

This completes the proof.

\section{Remark:}

1) $\operatorname{Sc}\left(C_{v}\left(K_{4}\right)_{2} ; x\right)=90 x+54 x^{2}$.

2) $S c^{*}\left(C_{v}\left(K_{4}\right)_{2} ; x\right)=162 x+81 x^{2}$.

Corollary 2.2.2: For $p \geq 2$, then we have:

1) $S c\left(C_{v}\left(K_{4}\right)_{p}\right)=3 p\left(4 p^{2}+9 p-1\right)$.

2) $S c^{*}\left(C_{v}\left(K_{4}\right)_{p}\right)=6 p\left(4 p^{2}+6 p-1\right)$

Corollary 2.2.3: For $p \geq 2$, then we have:

1) $\overline{S c}\left(C_{v}\left(K_{4}\right)_{p}\right)=\frac{2}{9}(12 p+23-32 /(3 p+1))$.

2) $\overline{S c^{*}}\left(C_{v}\left(K_{4}\right)_{p}\right)=\frac{8}{9}(6 p+7-23 / 2(3 p+1))$.

\section{Some Properties of the Coefficients of Schultz and Modified Schultz Polynomials}

A finite sequence $\left(a_{1}, a_{2}, \cdots, a_{h}\right)$ of $h$ positive integers is coefficients of polynomial $P(x)=\sum_{i=1}^{h} a_{i} x^{i}$. Then:

1) The polynomial $P(x)$ is called $j$-unimodal if, for some index $j$, $a_{1} \leq a_{2} \leq \cdots \leq a_{j} \geq a_{j+1} \geq \cdots \geq a_{h}$ and it is strictly $j$-unimodal if the inequality holds without equalities.

2) The polynomial $P(x)$ is called monotonically increasing (or monotonically decreasing) if, $a_{i} \leq a_{i+1}$ or $a_{i} \geq a_{i+1}$, respectively, for all $1 \leq i \leq h$ and it is strictly-increasing or strictly-decreasing respectively if the inequalities hold without equalities.

3) The polynomial $P(x)$ is called palindromic if $a_{i}=a_{h-i+1}$, for all $1 \leq i \leq h$ and is called semi-palindromic if $a_{j}=a_{h-j+1}, 1+i \leq j \leq h-i$ and for all $1 \leq i \leq h-2$.

4) The polynomial $P(x)$ is called troubled if $a_{i} \neq a_{i+1}$, for all $1 \leq i \leq h$.

5) The polynomial $P(x)$ is called equality if $a_{i}=a_{i+1}$, for all $1 \leq i \leq h$ and is called semi-equality if $a_{i}=a_{i+1}$ for some values $i$.

The following Table 3 shows the properties of polynomials coefficients of Schultz and modified Schultz of $C_{v}\left(C_{4}\right)_{p}$ and $C_{v}\left(K_{4}\right)_{p}$.

\section{Conclusion}

In this paper, we obtained the general formulas of polynomials for Schultz and modified Schultz polynomials of operation vertex identification chain for square and complete square graphs and indices of its. Also, we discuss some properties of the coefficients of these polynomials. 
Table 3. The some properties of the coefficients of $C_{v}\left(C_{4}\right)_{p}$ and $C_{v}\left(K_{4}\right)_{p}, p \geq 3$.

\begin{tabular}{cccccc}
\hline \multicolumn{1}{c}{ Some Properties } & & & & \\
$\begin{array}{l}\text { Polynomials } \\
\text { of types graphs }\end{array}$ & Property 1 & Property 2 & Property 3 & Property 4 & Property 5 \\
\hline$S c\left(C_{v}\left(C_{4}\right)_{p} ; x\right)$ & Satisfy at $j=2$ & Not satisfy & Not satisfy & Satisfy & Not satisfy \\
$S c^{*}\left(C_{v}\left(C_{4}\right)_{p} ; x\right)$ & Satisfy at $j=2$ & Not satisfy & Not satisfy & Satisfy & Not satisfy \\
$S c\left(C_{v}\left(K_{4}\right)_{p} ; x\right)$ & Satisfy at $j=2$ & Not satisfy & Not satisfy & Satisfy & Not satisfy \\
$S c^{*}\left(C_{v}\left(K_{4}\right)_{p} ; x\right)$ & Satisfy at $j=2$ & Not satisfy & Not satisfy & Satisfy & Not satisfy \\
\hline
\end{tabular}

\section{Acknowledgements}

This paper is has supported from Master's thesis for the student Mahmood M. A. from college computer sciences and mathematics, University of Mosul, Republic of Iraq.

\section{Conflicts of Interest}

The authors declare no conflicts of interest regarding the publication of this paper.

\section{References}

[1] Chartrand, G. and Lesniak, L. (2016) Graphs and Digraphs. 6th Edition, Wadsworth and Brooks/Cole, California.

[2] Buckley, F. and Harary, F. (1990) Distance in Graphs. Addison-Wesley, Longman.

[3] Su, G. and Xu, L. (2015) Topological Indices of the Line Graph of Subdivision Graphs and Their Schur-Bounds. Applied Mathematics and Computation, 253, 395-401. https://doi.org/10.1016/j.amc.2014.10.053

[4] Schultz, H.P. (1989) Topological Organic Chemistry. 1. Graph Theory and Topological Indices of Alkanes. J. Chem. Inf. Comput. Sci, 29, 227-228. https://doi.org/10.1021/ci00063a012

[5] Klavžar, S. and Gutman, I. (1997) Wiener Number of Vertex-Weighted Graphs and a Chemical Application. Discrete Applied Mathematics, 80, 73-81. https://doi.org/10.1016/S0166-218X(97)00070-X

[6] Ahmed, M.A. and Haitham, N.M. (2017) Schultz and Modified Schultz Polynomials of Two Operations Gutman's. International Journal of Enhanced Research in Science, Technology \& Engineering, 6, 68-74.

[7] Ahmed, M.A. and Haitham, N.M. (2019) Schultz and Modified Schultz Polynomials of Some Cog-Special Graphs. Open Access Library Journal, 6, 1-13. https://doi.org/10.4236/oalib.1105625

[8] Ali, A. (2019) Computing the Schultz Polynomials and Indices for Ladder Related Graphs. Proyecciones Journal of Mathematics, 39, 1081-1092. https://doi.org/10.22199/issn.0717-6279-2019-05-0070

[9] Haneen, K.A. (2017) Schultz Index, Modified Schultz Index, Schultz Polynomial and Modified Schultz Polynomial of Alkanes. Global Journal of Pure and Applied 
Mathematics, 13, 5827-5850.

[10] Mirajkar, K.G. and Doddamani, B.R. (2017) Schultz Polynomial and Their Topological Indices of Bottleneck Graphs. International Journal of Current Advanced Research, 6, 8312-8318.

[11] Wang, S., Farahani, M.R., Kanna, M.R.R. and Kumar, R.P. (2016) Schultz Polynomials and Their Topological Indices of Jahangir Graphs $J_{2, \mathrm{~m}}$. Applied Mathematics, 7, 1632-1637. https://doi.org/10.4236/am.2016.714140 